\title{
Desenvolvimento de formas farmacêuticas semissólidas contendo o extrato aquoso obtido das cascas do Anacardium occidentale L. e realização do estudo de estabilidade acelerado
}

RESUMO I Introdução: A Anacardium occidentale (cajueiro), é uma árvore tropical frutífera nativa do Brasil. Suas cascas são ricas em taninos, com ação anti-inflamatória e cicatrizante. Objetivo: Desenvolver formulações semissólidas: gel de carbopol, creme e pomada de lanovaselina contendo o extrato aquoso das cascas de Anacardium occidentale nas concentrações de 2,5\%, 5 \% e 7 \%, e realizar estudos de estabilidade acelerada, avaliando as características organolépticas, $\mathrm{pH}$, viscosidade e densidade relativa, nos tempos $\mathrm{T}(0)$ na data da produção, em temperatura ambiente, e T(30), T(60) e T(90) dias em estufa à $40^{\circ} \mathrm{C}$, afim de determinar a formulação mais estável. Resultados e conclusões: A formulação de creme apresentou melhor estabilidade em todas as concentrações do extrato e quanto a todos os parâmetros avaliados, no entanto, é necessário um estudo posterior para a padronização das formulações.

Palavras-chaves: Plantas medicinais; Estabilidade de medicamentos; Anacardium.

\begin{abstract}
Introduction: The Anacardium occidentale (cashew tree) is a tropical fruit tree native to Brazil. Their peels are rich in tannins, with anti-inflammatory and healing action. Aim: To develop semi-solid formulations: carbopol gel, cream and ointment containing the aqueous extract of the barks of Anacardium occidentale at concentrations of $2.5 \%, 5 \%$ and $7 \%$, and to perform accelerated stability studies, evaluating the organoleptic characteristics, $\mathrm{pH}$, viscosity and relative density, at $\mathrm{T}(0)$ times at the production date, at room temperature, and $\mathrm{T}(30), \mathrm{T}(60)$ and $\mathrm{T}(90)$ days at $40^{\circ} \mathrm{C}$ in order to determine the most stable. Results and conclusions: The cream formulation presented better stability at all concentrations of the extract and for all the evaluated parameters, however, a further study is necessary for the standardization of the formulations.
\end{abstract}

Keywords: Medicinal plants; Drug Stability; Anacardium.

RESUMEN | Introducción: el Anacardium occidentale (anacardo) es un árbol frutal tropical originario de Brasil. Sus cáscaras son ricas en taninos, con acción antiinflamatoria y curativa. Objetivo: Desarrollar formulaciones semisólidas: gel de carbopol, crema y pomada que contengan el extracto acuoso de las cortezas de Anacardium occidentale en concentraciones de $2.5 \%, 5 \%$ y $7 \%$, y realizar estudios de estabilidad acelerados, evaluando las características organolépticas, $\mathrm{pH}$, viscosidad y densidad relativa, en T (0) veces a la fecha de producción, a temperatura ambiente, y T (30), T (60) y T (90) días a $40^{\circ} \mathrm{C}$ para determinar el máximo estable Resultados y conclusiones: La formulación en crema presentó una mejor estabilidad en todas las concentraciones del extracto y para todos los parámetros evaluados, sin embargo, es necesario un estudio adicional para la estandarización de las formulaciones.

Palabras claves: Plantas medicinales; Estabilidad de medicamentos; Anacardio.

\section{Thaiane Vasconcelos Carvalho}

Farmacêutica. Residente Multiprofissional em Urgência e Emergência pela Santa Casa de Misericórdia de Sobral.

\section{Rildon Alves da Costa}

Farmacêutico. Coordenador da Farmácia Escola do Centro Universitário INTA (UNINTA).

\section{Ana Beatriz Melo Guimarães}

Farmacêutica. Residente Multiprofissional em Urgência e Emergência pela Santa Casa de Misericórdia de Sobral.

\section{lara Laís Lima de Sousa}

Fisioterapeuta. Especialista em Urgência e Emergência. Mestranda em Ciências da Saúde (UFC).

\section{Kauanny Gomes Gonçalves}

Enfermeira. Especialista em Urgência e Emergência.

\section{Wilcare de Medeiros Cordeiro Nascimento}

Farmacêutica/Mestre. Docente do Curso de farmácia do Centro Universitário INTA (UNINTA). 
$\longrightarrow$ Anacardium occidentale Liin, árvore tropical frutífera conhecida como cajueiro, de grande importância para uso medicinal², pertencente à família Anacardiaceae e ao bioma Caatinga, está inclusa na Relação Nacional de Interesse ao Sistema Único de Saúde - SUS (RENISUS) ${ }^{1}$, encontrando-se amplamente distribuída nas regiões Norte e Nordeste ${ }^{3}$.

Estudos científicos apresentam comprovadamente inúmeras atividades biológicas do cajueiro, envolvendo fruto, pseudofruto, folhas, flores e cascas do caule, tais como: atividade antimicrobiana de largo espectro ${ }^{3,4,5}$; antifúngica ${ }^{3}$; antioxidante ${ }^{2}$; giardicida ${ }^{7}$; ação anti-inflamatória e cicatrizante ${ }^{8,9}$; atividade hipoglicemiante e moluscicida ${ }^{10}$.

No Brasil, todos os produtos cosméticos tem a obrigatoriedade de indicar o prazo de validade nas embalagens, estabelecido na Resolução 79/00 e suas atualizações, e Lei 8.0178/90 do Código de Defesa do Consumidor. Dessa forma, os testes de estabilidade acelerada determinam mudanças físicas passíveis nas amostras, armazenadas em condições de estresse para prever o prazo de validade estimado e a qualidade dos produtos desenvolvidos ${ }^{6}$.

Diante da necessidade de ampliar as pesquisas relacionadas ao desenvolvimento de fitoterápicos com o Anacardium occidentale, planta amplamente utilizada de forma empírica na região Nordeste, buscou-se como objetivo da pesquisa desenvolver formulações de gel de carbopol, creme aniônico e pomada de lanovaselina contendo o extrato aquoso das cascas do Anacardium occidentale L. nas concentrações 2,5\%, $5 \%$ e $7 \%$, e compará-las através de testes de estabilidade acelerada.

\section{MÉTODO}

Trata-se de uma pesquisa de caráter experimental, com estudo primário, ana-

\section{6}

lítico, prospectivo, de abordagem qualitativa e quantitativa, realizada na Farmácia Escola do Centro Universitário INTA (UNINTA), na cidade de Sobral, Ceará, durante o período de abril a outubro de 2017.

Estudos científicos apresentam comprovadamente inúmeras atividades biológicas do cajueiro, envolvendo fruto, pseudofruto, folhas, flores e cascas do caule, tais como: atividade antimicrobiana de largo espectro ${ }^{3}$, 4, 5; antifúngica ${ }^{3}$; antioxidante $^{2}$; giardicida $^{7}$; ação anti-inflamatória e cicatrizante 8,9; atividade hipoglicemiante e moluscicida $^{10}$.
Preparação do Extrato

Foram utilizadas as cascas do caule do cajueiro (Anacardium occidentale), coletadas em maio de 2017, no horto de plantas medicinais e zona de bioprospecção do UNINTA, as quais passaram por um processo de beneficiamento primário com lavagem em água corrente, secagem em estufa a $40^{\circ} \mathrm{C}$, trituração em forrageira utilizando malha de $10 \mathrm{~mm}$, e preparação do extrato aquoso proposto pelo Núcleo de Fitoterapia do Estado do Ceará (NUFITO). Com o extrato padrão, foi realizado um teste para reconhecimento de taninos mediante as normas da Sociedade Brasileira de Farmacognosia, utilizando os reagentes: gelatina, cloreto férrico e acetato de chumbo.

Desenvolvimento das Formulações

Foram produzidas três formulações de uso tópico: gel de carbopol, creme aniônico e pomada de lanovaselina, de acordo com a Farmacopeia Brasileira, contendo o extrato nas concentrações de $2,5 \%, 5 \%$ e $7 \%$, em três lotes para cada uma, e uma amostra de cada formulação sem a presença do extrato, utilizada como branco.

Estudo de estabilidade acelerado

O estudo de estabilidade acelerado das formulações desenvolvidas foi realizado em triplicata, nos tempos: T 0 (zero), T 30 (trinta), T 60 (sessenta) e T 90 (noventa) dias. Considerando-se como tempo 0 (zero), o momento do ensaio realizado após a manipulação das formulações. As amostras foram mantidas em estufa na temperatura de $40^{\circ} \mathrm{C}$.

Avaliação das características físico-químicas

- Características organolépticas 
(aspecto, cor e odor);

- $\quad \mathrm{pH}$ (utilizando um potenciômetro calibrado com solução tampão pH 4,0 e 7,0, através da leitura de uma suspensão a 10 $\%$ de cada amostra das formulações);

- Viscosidade (através de um viscosímetro Brookfield digital, com spindle $\mathrm{n}^{\circ} 3$ a 4 );

- Densidade relativa (utilizando um picnômetro de metal), através da fórmula:

$$
d=\frac{P 2-P 0}{P 1-P 0}
$$

Onde: $d$ = densidade;

$\mathrm{P0}=$ peso do picnômetro vazio;

$\mathrm{P} 1$ = peso do picnômetro com água purificada;

P2 = peso do picnômetro com as amostras.

Todas as formulações foram analisadas com a embalagem primária que estaria disponível para entrega ao consumidor quando finalizadas, a fim de que as análises e resultados fossem mais precisos e confiáveis. Os testes aplicados tiveram como objetivo verificar a estabilidade dos produtos finais obtidos e estão descritos no Guia de Estabilidade de
Produtos Cosméticos, fundamentados na Farmacopeia Brasileira. Foram utilizados os parâmetros de média e desvio padrão para determinar a média dos valores obtidos nos testes.

\section{RESULTADOS}

Os testes de reconhecimento de taninos no extrato aquoso das cascas de Anacardium occidentale foram positivos em todos os reagentes, sendo identificada a presença de taninos (formação de precipitados com gelatina), taninos hidrolisáveis (coloração azul com cloreto férrico) e taninos condensados (formação de precipitados com acetato de chumbo).

Quanto ao aspecto, o gel apresentou-se homogêneo e brilhante inicialmente (T0), e com o passar do tempo (T30, T60 e T90) adquiriu um aspecto grumoso em todas as amostras e concentrações $(2,5$ $\%, 5 \%$ e $7 \%$ ). O creme por sua vez, manteve-se homogêneo e brilhante em todas as amostras e concentrações (2,5\%, 5\% e $7 \%$ ) durante todo o período do estudo. Já a pomada de lanovaselina apresentou-se homogênea, brilhante e oleosa. No entanto, em todas as concentrações $(2,5 \%, 5 \%$ e $7 \%)$, desde o T0, notou-se a separação de fases tanto em temperatura ambiente, como quando levada à estufa a $40^{\circ} \mathrm{C}$, necessitando de homogeneização.

As formulações adquiriram uma cor variável, porém característica à presença do extrato. E avaliando o odor, o gel apresentou odor forte das cascas do cajueiro, enquanto o creme e a pomada mantiveram seus odores característicos semelhantes ao padrão.

$\mathrm{Na}$ avaliação do $\mathrm{pH}$, apesar da queda do valor apresentado pelas médias dos lotes em suas respectivas concentrações, nota-se que o desvio padrão foi leve (Tabela 1), não havendo uma discrepância nos valores obtidos.

As formulações de gel de carbopol contendo o extrato a 7\% obtiveram maior viscosidade que as formulações com extrato a 2,5 e $5 \%$.

Já as formulações de creme obtiveram valores de viscosidade mais estáveis, porém, apesar da pequena diferença de variação entre a viscosidade dos cremes nas diferentes concentrações, observa-se uma leve redução da viscosidade conforme o aumento da concentração do extrato. A amostra de creme base (branco) permaneceu estável em todos os tempos, e mesmo sob a temperatura de $40^{\circ} \mathrm{C}$ em estufa manteve-se com os mesmos valores de viscosidade, mostrando que o extrato provoca variações na viscosidade da fórmula.

Tabela 1 - Média e desvio padrão do pH das formulações de gel, creme e pomada contendo o extrato de A. occidentale, nos tempos T(0), T (30), T (60) e T (90) dias.

\begin{tabular}{|c|c|c|c|c|}
\hline GEL & pH (T0) & pH (T30) & $\mathrm{pH}(\mathrm{T} 60)$ & pH (T90) \\
\hline $2,5 \%$ & $6,08 \pm 0,017$ & $5,90 \pm 0,01$ & $5,73 \pm 0,03$ & $5,56 \pm 0,67$ \\
\hline $5 \%$ & $5,75 \pm 0,24$ & $5,51 \pm 0,11$ & $5,28 \pm 0,07$ & $5,05 \pm 0,22$ \\
\hline $7 \%$ & $5,87 \pm 0,13$ & $5,49 \pm 0,13$ & $5,18 \pm 0,36$ & $4,73 \pm 0,44$ \\
\hline CREME & $\mathrm{pH}(\mathrm{TO})$ & pH (T30) & $\mathrm{pH}(\mathrm{T} 60)$ & $\mathrm{pH}(\mathrm{T} 90)$ \\
\hline $2,5 \%$ & $4,28 \pm 0,10$ & $3,56 \pm 0,04$ & $3,42 \pm 0,05$ & $3,35 \pm 0,03$ \\
\hline $7 \%$ & $4,52 \pm 0,81$ & $3,65 \pm 0,08$ & $3,52 \pm 0,04$ & $3,32 \pm 0,07$ \\
\hline POMADA & $\mathrm{pH}(\mathrm{TO})$ & $\mathrm{pH}(\mathrm{T} 30)$ & $\mathrm{pH}(\mathrm{T} 60)$ & $\mathrm{pH}(\mathrm{T} 90)$ \\
\hline $2,5 \%$ & $7,58 \pm 0,25$ & $5,84 \pm 0,52$ & $5,62 \pm 0,45$ & $5,50 \pm 0,44$ \\
\hline $5 \%$ & $7,02 \pm 0,39$ & $5,32 \pm 0,11$ & $5,20 \pm 0,08$ & $5,07 \pm 0,05$ \\
\hline
\end{tabular}

Valores expressos em média \pm desvio padrão. 
As formulações de pomada de lanovaselina tanto em branco (formulação base), como após a incorporação do extrato em todas as concentrações não obtiveram estabilização, não sendo possível realizar a avaliação da viscosidade.

Outro parâmetro farmacopeico importante para o controle de qualidade das formulações semissólidas, é a densidade relativa, que foi avaliada em cada formulação, e calculada a média e desvio padrão (Tabela 2). seu efeito adstringente justifica a ação cicatrizante e anti-inflamatória, pois os mesmos precipitam as proteínas dos tecidos lesados, formando um revestimento protetor que favorece a sua reparação, diminuindo a permeabilidade e exsudação da ferida ${ }^{11}$.

A aplicação de ensaios organolépticos permite avaliar de forma rápida as condições em que se encontram os produtos obtidos, através de comparações feitas com a forma farmacêutica base, conhecida também como padrão. dade ótima para a maioria dos fármacos hidrolisáveis, a faixa de $\mathrm{pH}$ ácido deve-se estar ente 4 e 6.

$\mathrm{Na}$ avaliação da estabilidade de formulações contendo o extrato do cajueiro, o $\mathrm{pH}$ inicial era 6,0, compatível com a utilização tópica, no entanto, com o armazenamento a $60^{\circ} \mathrm{C}$, houve uma redução no $\mathrm{pH}$, indicando que o calor promove a decomposição do produto. O estudo ainda aponta a escurecimento gradual e mudança no odor característico das formulações ${ }^{18}$.

Tabela 2 - Média e desvio padrão da densidade das formulações de gel, creme e pomada contendo o extrato de A. occidentale, nos tempos T(0), T (30), T (60) e T (90) dias.

\begin{tabular}{|c|c|c|c|c|}
\hline GEL & Densidade (T0) & Densidade (T30) & Densidade (T60) & Densidade (T90) \\
\hline $5 \%$ & $0,85 \pm 0,03$ & $1,00 \pm 0,03$ & $0,87 \pm 0,00$ & $0.97 \pm 0,00$ \\
\hline $7 \%$ & $0,94 \pm 0,06$ & $0,96 \pm 0,04$ & $0,89 \pm 0,01$ & $0,91 \pm 0,00$ \\
\hline CREME & Densidade (T0) & Densidade (T30) & Densidade (T60) & Densidade (T90) \\
\hline $7 \%$ & $0,97 \pm 0,00$ & $1,00 \pm 0,00$ & $1,00 \pm 0,00$ & $0,89 \pm 0,03$ \\
\hline POMADA & Densidade (T0) & Densidade (T30) & Densidade (T60) & Densidade (T90) \\
\hline $2,5 \%$ & $0,79 \pm 0,07$ & $0,88 \pm 0,00$ & $0,87 \pm 0,00$ & $0,82 \pm 0,00$ \\
\hline $5 \%$ & $0,85 \pm 0,01$ & $0,90 \pm 0,01$ & $0,89 \pm 0,01$ & $0,81 \pm 0,00$ \\
\hline
\end{tabular}

Valores expressos em média \pm desvio padrão.

Não há na Farmacopeia Brasileira ou qualquer outro compêndio oficial nacional as especificações de densidade relativa para estas formulações, porém, o extrato aquoso das cascas de Anacardium occidentale não provocou alterações na densidade das formulações. Em média, as formulações de creme apresentaram maior densidade relativa, mas com uma mínima diferença entre as formulações de gel e pomada.

\section{DISCUSSÕES}

Segundo um levantamento etnobotânico realizado na Caatinga do Nordeste do Brasil, 500g de amostra de cascas secas de Anacardium occidentale possuem um teor de $65,19 \%$ para a presença de taninos. O
Segundo Shimabuku, os cremes não apresentam nenhuma alteração em suas características organolépticas. De modo que em comparação a outras formas farmacêuticas, possuem uma boa consistência e aceitabilidade ${ }^{13}$.

De acordo com o estudo feito por Mascarenhas, a pomada de lanovaselina contendo o extrato aquoso de Jucá (Caesalpinea ferrea) também apresentou separação de fases entre a forma farmacêutica e o extrato aquoso recorrente em estufa e em temperatura ambiente ${ }^{12}$.

Tanto a temperatura quanto o $\mathrm{pH}$ são fatores que afetam a estabilidade de fármacos propensos à decomposição hidrolítica ${ }^{13}$. Portanto, faz-se necessária a refrigeração para muitas preparações sujeitas a hidrólise, e para obter-se uma estabili-
Apesar do registro da viscosidade pelo aparelho, há uma oscilação dos valores, podendo ser associado à formação de bolhas na fórmula, dificultando a determinação de um padrão de resultados e/ou modificações nos mesmos conforme os tempos na estufa. Em seus estudos, Mascarenhas ${ }^{12}$ relata que não foi possível a leitura da viscosidade em gel de carbopol contendo o extrato de Jucá, também por consequência da formação de bolhas na fórmula, enquanto Costa ${ }^{15}$ que realizou experimentos com um gel contendo extrato de Noni, afirma que a viscosidade do gel aumenta conforme o aumento da concentração do extrato.

Contudo, a queda da viscosidade 
das formulações contendo o extrato a $7 \%$ ao longo do tempo, pode ser justificada pela temperatura da estufa, pois de acordo com a Farmacopeia Brasileira, o aumento da temperatura, aumenta a energia cinética média das moléculas, diminui (em média) o intervalo de tempo que as moléculas passam umas junto das outras, tornando-se menos efetivas as forças intermoleculares e diminuindo a viscosidade ${ }^{16}$.

Essa mesma característica foi encontrada nos experimentos de $\mathrm{Pa}$ rente $^{14}$, Mascarenhas ${ }^{12}$, que também mostraram que os resultados de viscosidade são inversamente proporcionais às concentrações do extrato. Contudo, faz-se necessária a realização de novos testes em novas amostras formuladas para obtenção de resultados mais concretos e estabilizados.
A avaliação da viscosidade por sua vez, auxilia na determinação da consistência ou fluidez apropriada e indica a adequação da estabilidade das formulações ${ }^{14}$.

Segundo Oliveira, a determinação da viscosidade nos testes de estabilidade não está relacionada com valores absolutos e exatos de viscosidade, mas sim, com a avaliação das possíveis alterações desta propriedade com o passar do tempo, notando-se, portanto, que o creme em estudo não apresentou variações exacerbadas ${ }^{17}$.

No entanto, não foi possível avaliar esse parâmetro todas as amostras, visto que durante o experimento fez-se necessário mudança da velocidade de cisalhamento do aparelho Brookfield, que segundo manual, para obter-se um resultado mais fidedigno, é necessário que o mesmo esteja ajustado para até ou mais próximo de $50 \%$ do cisalhamento.

\section{CONCLUSÃO}

Para o desenvolvimento de formulações semissólidas é necessário a realização do controle de qualidade e estabilidade, visando a garantia da segurança e eficácia do uso tópico dos produtos pelo consumidor. Neste estudo, a formulação de creme desenvolvida obteve os melhores resultados em relação ao gel e a pomada diante dos testes de estabilidade acelerada realizados. Contudo, estes testes não são suficientes para assegurar a qualidade total dos produtos, sendo necessária a realização de mais estudos pré-clínicos e microbiológicos, a fim de assegurar um produto final de qualidade.

\section{Referências}

1. Viana, GSB; Leal, LKAM; Vasconcelos, SMM. Plantas Medicinais da Caatinga: Atividades Biológicas e Potencial Terapêutico. $1^{\mathrm{a}}$. ed. Fortaleza: Expressão Gráfica e Editora, 2013.

2. Santos, FO. Atividades Biológicas de Anacardium occidentale (Linn). Patos: Universidade Federal de Campina Grande, 2011. Dissertação de Mestrado em Sistemas Agrossilvipastoris no Semi-árido.

3. Silva, RA. Ação antimicrobiana de Anacardium occidentale L.: potencial biotecnológico na geração de produtos anticárie. São Luís: Rede Nordeste de Biotecnologia - RENORBIO, 2012. Tese de Doutorado em Biotecnologia.

4. Chaves et al. Fenóis totais, atividade antioxidante e constituintes químicos de extratos de Anacardium occidentale L., Anacardiaceae. Revista Brasileira de Farmacognosia. 2010; 20; 106-112 (1)

5. Araújo et al. Concentração mínima bactericida do extrato do cajueiro sobre bactérias do biofilme dental. Pesquisa Brasileira em HYPERLINK "https://www.google.com.br/url?sa=t\&rct=j\&q=\&esrc=s\&source=web\&$\mathrm{cd}=1 \& \mathrm{cad}=$ rja\&uact $=8 \&$ ved $=0$ ahUKEwjZ_auMgufOAhXLE5AKHcNuBnkQFggcMAA\&url=http $\% 3 \mathrm{~A} \% 2 \mathrm{~F} \% 2 \mathrm{Fscholar}$.google.com.br\%2Fcitations $\%$ 3Fuser\%3DimmViDUAAAAJ\%26hl\%3Dpt-BR\&usg=AFQjCNFGs8NyWEWJg94Ades18KIwJQn_3w\&bvm=bv.131286987,d.Y2I"Odontopediatria HYPERLINK "https://www.google.com.br/url?sa=t\&rct=j\&q=\&esrc=s\&sour$c e=$ web \&cd $=1 \& c a d=r j a \& u a c t=8 \& v e d=0$ ahUKEwjZ_auMgufOAhXLE5 AKHcNuBnkQFggcMAA\&url=http $\% 3 \mathrm{~A} \% 2 \mathrm{~F} \% 2 \mathrm{Fscholar}$.google.com br\%2Fcitations\%3Fuser\%3DimmViDUAAAAJ\%26hl\%3Dpt-BR\&usg=AFQjCNFGs8NyWEWJg94Ades18KIwJQn_3w\&bvm=bv.131286987,d.Y2I" e Clínica Integrada. 2009; 9; 187-191 (2).

6. Sousa, BC. Anacardium occidentale: Avaliação do efeito fotoprotetor e conservante em preparações cosméticas. Recife: Universidade Federal de Pernambuco, 2008. Dissertação de mestrado em Ciências Farmacêuticas.

7. Filho, FAT. et al. Estudo e padronização de extratos de Anacardium occidentale L. na pesquisa e desenvolvimento de fitoterápicos giardicidas. Caderno de Pesquisa. 2013; 20; 7-15.

8. Schirato, GV. et al. 0 polissacarídeo do Anacardium occidentale L. na fase inflamatória do processo cicatricial de lesões cutâneas. Ciência Rural. 2006;
36; 149-154, (1).

9. Vasconcelos, MS. Atividades antioxidante, anti-inflamatória e cicatrizante do caju (Anacardium occidentale L.). Fortaleza: Universidade Federal do Ceará, 2011. Dissertação de mestrado em Bioquímica.

10. Santos, FA. et al. Anacardium occidentale Linn. (cajueiro). Plantas Medicinais da Caatinga: Atividades Biológicas e Potencial Terapêutico. $1^{\text {a }}$ ed. Fortaleza: Expressão Gráfica Editora ; 2013. p. 61-92.

11. Martins, PS. et al. Comparison between phytotherapics on equine wound healing. Archives of Veterinary Science. 2003; 8; 1-7; (2).

12. Mascarenhas, LB. Desenvolvimento e estudo de estabilidade acelerado de uma formulação tópica com extrato de Caesalpinea ferrea. Sobral: Faculdades INTA, Instituto Superior de Teologia Aplicada, 2015. Trabalho de Conclusão de Curso em Farmácia.

13. Shimabuku et al. Avaliação da qualidade de cremes dermatológicos manipulados na cidade de Marilia, SP. Colloquium Vitae. 2009; 1; 30-37, (1).

14. Parente, DS. Desenvolvimento de fitoterápicos de uso tópico a partir do extrato hidroalcoólico das folhas do noni (Morinda citrifolia L.) e realização do controle de qualidade físico-químico. Sobral: Faculdades INTA, Instituto Superior de Teologia Aplicada, 2015. Trabalho de Conclusão de Curso em Farmácia. 15. Costa, RA. Desenvolvimento de formulações semissólidas e realização de teste de estabilidade preliminar de formas farmacêuticas obtidas a partir do extrato hidroalcoólico das folhas do noni (Morinda citrifolia L.), Sobral: Faculdades INTA, Instituto Superior de Teologia Aplicada, 2016. Trabalho de Conclusão de Curso em Farmácia.

16. Brasil. Formulário Nacional da Farmacopeia Brasileira. \{publicação na web\}; 2012 ; acesso em: 12 set. 2016. Disponível em: http://www.anvisa.gov. br/hotsite/farmacopeiabrasileira/arquivos/2012/FNFB\%202_Revisao_2_COFAR_setembro_2012_atual.pdf.

17. Oliveira, AZZM. Desenvolvimento de formulações cosméticas com ácido hialurónico. Porto: Faculdade de Farmácia, Universidade do Porto, 2009. Dissertação de Mestrado em Tecnologia Farmacêutica.

18. Gonçalves, GMS. Antimicrobial Effect of Anacardium Occidentale Extract and Cosmetic Formulation Development. 2012; 55; 843-850; (6). 